\title{
Edge effects on the fluttering characteristics of freely falling planar particles
}

\author{
Luis Blay Esteban, ${ }^{*}$ John Shrimpton,${ }^{\dagger}$ and Bharathram Ganapathisubramani ${ }^{\ddagger}$ \\ Faculty of Engineering and the Environment, \\ University of Southampton, University Rd, Southampton SO17 1BJ, UK
}

(Dated: May 21, 2018)

\begin{abstract}
The effect of particle edge geometry on the descent motion of free falling planar particles is examined through experiments. Various planar particles, such as disk and polygons, with identical frontal areas $\left(A_{p}\right)$ and different number of edges (or perimeter) are used. All particles are designed such that their values of Galileo number $(G)$ and dimensionless moment of inertia $\left(I^{*}\right)$ correspond to the previously identified fluttering regime of particle motion. Several modes of secondary motion are observed for the same particle and conditions, and these are not equally probable. This probability depends on the particle shape. Disks and heptagons were found to prefer a 'planar zig-zag' behaviour. These planar motions are composed of gliding sweeps and turning sections. As the number of sides in the polygon decreases, i.e. for hexagons and pentagons, the trajectory transition to a more three-dimensional form. These trajectories were found to be restricted to one plane per swing but the subsequent swings are in other planes. Further decrease in number of sides to a square results in the trajectories having a severe out-of-plane motion. These sub-regimes of particle motion within the fluttering regime are consistent with those reported for disks in previous studies. Based on this information, a new length scale that accounts for the frontal area of the particles and its edge geometry (i.e. perimeter) is proposed. This length scale represents the first approach to determine an equivalent disks for planar particles such that the phase digram in the Reynolds number $(R e)$ - dimensionless moment of inertia $\left(I^{*}\right)$ domain can be used to characterise the motion of planar particles with different frontal geometries. However further experiments covering other domains of the regime map are needed to verify its universality.
\end{abstract}

\footnotetext{
*lbe1g14@soton.ac.uk

† john.shrimpton@soton.ac.uk

‡g.bharath@soton.ac.uk
} 


\section{INTRODUCTION}

Aspherical particles, especially planar particles of different shapes and sizes, are ubiquitous in various environmental and industrial processes. Natural processes include pollen and seed dispersion in the atmosphere, Sabban and van Hout [15]; whereas industrial processes include pharmaceutical processing, Erni et al. [6] and the emission of contaminants from combustion processes as in Moffet and Prather [14].

The descent motion of freely falling particles is complex, even for classical simple geometries such as disks and rectangular plates. These particles show a variety of falling regimes that go from steady fall to tumbling, through fluttering and chaotic motion. The first phase diagram showing the falling scenarios of a disk was defined by Willmarth et al. [17] in the Reynolds number $(R e)$ and the dimensionless moment of inertia $\left(I^{*}\right)$ domain. The Reynolds number defined as $R e=V_{z} D / \nu$, where $V_{z}$ is the average settling velocity, $D$ is the diameter of the disk and $\nu$ is the kinematic viscosity of the fluid. The dimensionless moment of inertia as $I^{*}=I_{p} / \rho_{f} D^{5}$, where $I_{p}$ is the moment of inertia about the diameter of the disk and $\rho_{f}$ is the density of the fluid. They carried out a series of experiments and mapped the different falling regimes they observed: steady, fluttering and tumbling. They discussed that the phase diagram held for disks, as long as the aspect ratio $(\chi)$ defined as $\chi=D / h$ was large (where $h$ is the thickness of the disk). The same approach was later followed by Smith [16] to construct a new $R e-I^{*}$ phase diagram for falling plates.

Field et al. [8] identified a new falling regime within the phase diagram defined by Willmarth et al. [17] between flutter and tumbling regimes. This new regime was labelled as 'Chaotic' and has been investigated in more detail during the last decades, especially for two-dimensional rectangular plates. Belmonte et al. [4] increased $I^{*}$ in an experiment where the plate motion was constrained mechanically to two dimensions, defining the transition from fluttering to tumbling. Similarly, Andersen et al. [2] measured the instantaneous fluid forces from the two-dimensional plate accelerations. In Andersen et al. [1] they also used direct numerical simulations of the two-dimensional Navier-Stokes equations and a fluid force model to investigate the transition between periodic fluttering and periodic tumbling.

Fernandes et al. [7] performed an experimental investigation on the oscillatory motion of axisymmetric bodies in the range of $80<R e<330$, and $1.5<\chi<20$. They showed that as $\chi$ increases, the axial symmetry of the wake breaks and wake oscillations appear at smaller 
Reynolds numbers. They distinguished between thick $(\chi<6)$ and thin $(\chi>6)$ bodies by comparing the shedding frequency of these with a freely falling disk and a fixed body wake.

Zhong et al. [18] focused on thin disks, according with the definition of Fernandes et al. [7], and showed experimentally that disks with $\chi \geq 10$ exhibit paths that transition from 'planar zig-zag' to 'spiral' motion for a region in the phase diagram associated with fluttering motion. They discussed that this transition is determined by the relative particle to fluid inertia $\left(I^{*}\right)$. They also carried flow visualization and showed that the change in path style comes with a drastic change in the turbulent structures present in the wake. They discussed that the transition between these three sub-regimes, i.e. 'planar zig-zag', 'transitional' and 'spiral', is Reynolds number dependent. However, statistical information about the likeliness of these transitions to occur was not provided. Lee et al. [11] extended the work of Zhong et al. [18] by doing planar PIV measurements on the particle wake. They found that disks with moderate inertia followed a 'planar zig-zag' motion and with decreasing $I^{*}$ the particles transitioned to a 'spiral' motion. They also investigated the effect of initial tilt (up to $10 \mathrm{deg}$ ) and showed that this phenomena was present for all angles tested. On the other hand, Heisinger et al. [9] showed that the same disk (for a given "high" Reynolds number and dimensionless moment of inertia) can follow both types of motion; i.e. 'planar zig-zag' and 'spiral'.

Auguste et al. [3] extended the work of Fernandes et al. [7] regarding the influence of the particle aspect ratio $(\chi)$ on the particle dynamics. They questioned the influence of the disk aspect ratio for $\chi>10$ at low-to-moderate Reynolds number (typically $R e<300$ ) and found remarkable differences in the dynamics of disks for different $\chi$ values. In their study, the Reynolds number based on the average descent velocity of the disk was replaced by the Archimedes number $A r=\sqrt{(3 / 32)} U_{g} D / \nu$, based on the gravitational speed $U_{g}=$ $\sqrt{2\left|\rho_{p} / \rho_{f}-1\right| g h}$. This defines $A r$ as a true control parameter as oppose to $R e$, since the average descent velocity is not known a priori. They focused on the transition from 'Steady Vertical' (SV) motion to 'Zig-Zag' (ZZ) motion and found that for disks with significant inertia, the transition takes place over distances of hundreds of disk diameters before reaching a saturated state. They found a 'Hula-Hoop' (HH) falling style that is qualitatively similar to the 'spiral' motion reported in Zhong et al. [18]. However, in Auguste et al. [3], this transition corresponds to a SV - ZZ - HH scenario at much lower Reynolds numbers. They hypothesised that increasing $A r$, the planar symmetry of the wake and path might break, 
yielding twisted structures and three-dimensional paths in which the plane of the fluttering motion slowly rotates about the vertical axis.

Chrust et al. [5] focused on the dynamics of freely falling infinitely thin disks. They performed direct numerical simulations of the solid-fluid interaction and presented a parametric study on the effect of the Galileo number $\left(G=\sqrt{\left|\rho_{p} / \rho_{f}-1\right| g D^{3}} / \nu\right)$, which is an alternate form of $A r$, and the dimensionless mass $\left(m^{*}\right)$, being $m^{*}=\frac{m}{\rho_{f} D^{3}}$. The results obtained for low-to-moderate $G$ agree well with the aforementioned falling styles in Auguste et al. [3]. They also studied 'Three-dimensional states' and found similar falling styles for disks with $I^{*}=3.12 \times 10^{-3}$ and $R e=320$ as reported in Zhong et al. [18]. They showed that the saturated 'spiral' state was reached after a fall distance of $\approx 60 \mathrm{D}$. The distance travelled by the disk to reach the 'spiral' state greatly differs in both studies, suggesting that not only the dimensionless moment inertia but the Reynolds number affects the length of the transient effects. Following the same approach, Zhou et al. [19] also showed the coexistance of several drastically different modes of motion for oblate spheroids lying within the same parameter space.

Results from experimental research and direct numerical simulations focused on the dynamics of thin disks suggest that the falling motion of these particles is not unique even for a single particle falling in the same media and therefore should be examined through statistical analysis. In this work we measure the trajectory variation of a disk for a large number of realizations and compare it with the trajectories of other planar polygons.

Fewer studies are available for shapes other than disks or rectangular plates. List and Schemenauer [12] investigated the steady fall of different shapes including a hexagonal plate, a broad-branched crystal, a stellar crystal with plates and a dendrite. In addition to an increase of drag coefficient with Reynolds number (due to viscous effects), they observed small oscillations in the fall trajectories of hexagonal plate and broad-branched models. Jayaweera [10] studied experimentally the free fall behaviour of various planar particles within the same range of Reynolds number $(R e<50)$. All planar particles were manufactured with the same frontal area and thickness of a reference disk for comparison. They showed that the terminal velocity of hexagonal plates was practically the same of the equivalent disk while for the case of star-shape particles the differences in the terminal velocity were up to $25 \%$.

Moffatt [13] reviewed the results in Auguste et al. [3] and suggested three questions for future investigation regarding the dynamics of disks; (i) the effect of surface roughness and 
disk rim, (ii) the effect adding a hole in it and (iii) the effect of the edge geometry. In this paper, we aim to characterise the fluttering regime of the $R e-I^{*}$ phase diagram updated by Lee et al. [11] for different shaped particles as a first attempt to tackle question (iii) proposed in Moffatt [13]. We vary the particle shape keeping the particle frontal area $\left(A_{p}\right)$, $\chi$ and $\rho_{p}$ nearly constant and aim to understand if the sub-regimes within the flutter regime found for disks (Zhong et al. 18, Lee et al. 11 and Chrust et al. 5) also holds for other planar particles. We carry out experiments and measure the 3D trajectories of planar regular polygons, i.e. heptagons, hexagons, pentagons and squares with comparable dimensionless moments of inertia $\left(I^{*}\right)$ based on the frontal area of the particle. The reference particle is a disk manufactured to lie in the 'planar zig-zag' region $\left(G \approx 4000\right.$ and $\left.I^{*} \approx 5 \times 10^{-3}\right)$. The particle descent velocity and the degree of two-dimensionality of the trajectories are discussed. The degree of two-dimensionality is used to classify the particle trajectories in three subgroups: 'planar zig-zag' motion, 'transition' motion and 'spiral' motion. The findings are used to develop a new particle length scale that incorporates the degree of axisymmetry of the frontal geometry of the particle through the iso-perimetric quotient. This length scale allows us to use the original $R e-I^{*}$ phase diagram for disks but for the n-polygon particles investigated in here.

\section{EXPERIMENTAL FACILITY AND MEASUREMENT TECHNIQUE}

\section{A. Particles}

A single set of planar regular polygons are manufactured to have the same frontal area as the reference disk within the precision of the laser cutter $\pm 0.5 \mathrm{~mm}$. The particles were made of acrylic with a density of approximately $1.2 \mathrm{~g} / \mathrm{cm}^{3}$, frontal area of $4.4 \mathrm{~cm}^{2}$ and a thickness of $2 \mathrm{~mm}$. All particles were painted black for image processing. Table I summarizes the dimensions of the particles manufactured.

\section{B. Description of the facility}

The experimental design in figure 1(a) shows the water tank and digital camera set-up. The water tank was $0.8 \mathrm{~m}$ high and had a cross-section of $0.5 \times 0.5 \mathrm{~m}$. An active suction mechanism to hold the particles in place was attached to the tank structure. This was 
Disk Heptagon Hexagon Pentagon Square

\begin{tabular}{|c|c|c|c|c|}
\hline Symbol & 0 & $*$ & $\xi \xi$ & $i s$ \\
\hline Side $(\mathrm{cm})$ & - & 1.1 & 1.3 & 1.6 \\
\hline$h(\mathrm{~cm})$ & 0.2 & 0.2 & 0.2 & 0.2 \\
\hline$P(\mathrm{~cm})$ & 7.4 & 7.7 & 7.8 & 8 \\
\hline$A_{p}\left(\mathrm{~cm}^{2}\right)$ & 4.34 & 4.40 & 4.39 & 4.40 \\
\hline Mass (g) & 1.07 & 1.02 & 1.01 & 1.02 \\
\hline
\end{tabular}

TABLE I: Particle dimensions. $P$ refers to the particle perimeter.

horizontally positioned inside the water tank at a depth of $2 D$ bellow the water level so that entry and surface effects were avoided. This mechanism consists of a suction cap of $5 \mathrm{~mm}$ diameter connected to a square flat plate of $0.25 \times 0.25 \mathrm{~m}$ acting as a partially open lid. The suction cup was flushed into the submerged flat plate. Thus, when the particle was positioned the upper surface of the particle was in contact with both the suction cup and the plate. Therefore, the angle of the particle was the same as the submerged flat plate. The alignment of the submerged plate was carefully performed before filling the tank using a digital angle with an accuracy of 0.1 deg. Despite these considerations the particle might have been released with a marginally different angle in between particle side and incoming flow in each realization. However, the effect of initial tilt is known to only influence in the early stage of the particle descent, as shown in [11].

The open section between the glass walls and the edge of the flat plate is used to position the particles before each drop. The vacuum circuit is equipped with a release valve opened to the atmosphere that is used to drop the particles. Two cameras were used to capture two views of the falling particle and a diffused light source was used for illumination. The cameras were both focused on the mid-plane of the tank to minimize image distortion. Careful attention was paid to the camera alignment to make the two views orthogonal in order to ensure an accurate 3D trajectory reconstruction. 
a)

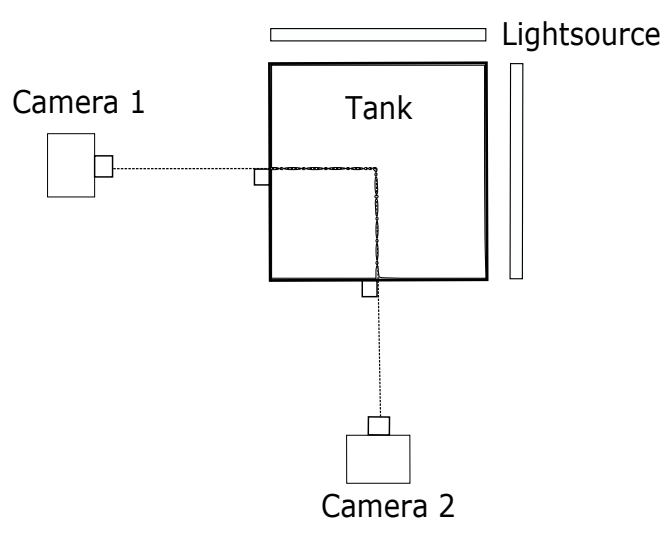

b)

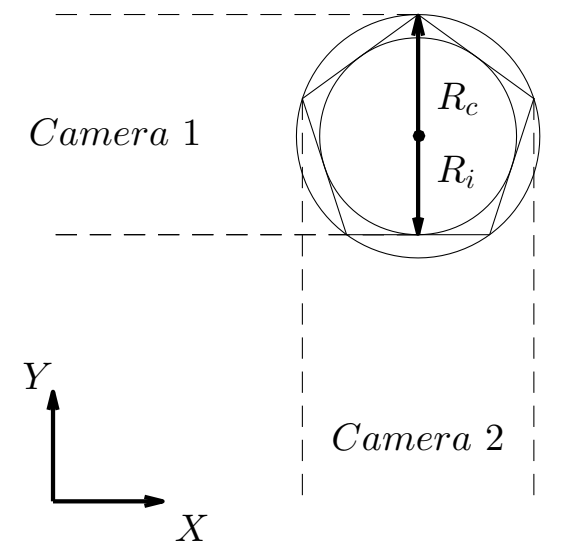

FIG. 1: Schematic of the camera and illumination set-up for trajectory reconstruction (a); top view of a pentagon positioned for maximum error in the center of gravity determination for the camera measuring the $\mathrm{Y}$ coordinate(b).

\section{Method}

All particles were held horizontal in their initial position by a suction cup smaller than their internal diameter and the drop position was centred to avoid wall effects during the particle fall. The initial tilt of the particle prior to the release was carefully checked. When the particle is released, trajectories were recorded at $60 \mathrm{fps}$. This frame-rate was sufficient to resolve the translational motion during all parts of the descent. In each frame the dark particle projection was recorded onto the white background and the position of the particle center of mass was obtained by locating the geometric center of each particle projection. The measured trajectories were smooth and a polynomial filter of 3rd order and frame length of 5 points was used to filter out high frequency noise.

A set of releases for a sphere falling in air was performed to verify the alignment of the set up. To account for image distortion due to light refraction we placed a square grid in the field of view of the cameras and we found a negligible image distortion within the interrogation area. The variance in the landing position of the sphere was interpreted as the uncertainty of the set up alignment. This was found to be at least two orders of magnitude smaller than the sphere diameter. This is in accordance with the uncertainty typically found in the literature, as in Heisinger et al. [9].

The process followed to obtain the particle center of mass did not add any uncertainty 
on the particle location for the case of a disk or any planar particle with an even number of sides. However, the fact that the edges of the planar particles are not easily identified by the cameras could add uncertainty to the determination of the center of gravity of particles with an odd number of sides, see fig 1 (b). The maximum possible uncertainty in the center of gravity location associated with this issue is half of the difference between the radius of the circumscribed and inscribed circle, being maximum for the pentagon $\left( \pm 0.02 \sqrt{A_{p}}\right)$.

To build a baseline from which to compare the motion of the planar particles a first set of 300 repeated realizations of the disk falling in water were performed. Since the study of the initial transient dynamics is out of the scope of this study the trajectories are studied after the particle reached a saturated state. The determination of the distance at which the particle motion is not influenced by the transient dynamics is non-trivial. Chrust et al. [5] showed that infinitely thin disks with $I^{*}=3.12 \times 10^{-3}$ and $G=300$ showed saturated paths at a vertical distance of $\approx 60 D$ from the release point, whereas Heisinger et al. [9] showed experimentally that for disks with $I^{*} \approx 3 \times 10^{-3}$ and $G \approx 5647$, the disk trajectory was saturated after a distance of $7 D$. In here, the effect of the initial release angle $\theta$ on the style of the particle descent and trajectory saturation is investigated for the square; since it could be the most affected particle by the initial conditions (being the least axisymmetric). An angle $\theta=6.5 \mathrm{deg}$ is chosen as a relatively large angle compared with the typical uncertainty $( \pm 0.01 \mathrm{deg})$ in the particle alignment and as a 'typical' angle during the particle descent. The angle of the particle is controlled by tilting the submerged flat plate, and measured prior to each particle drop. Two different initial particle orientations relative to the projected trajectory are tested; i.e. a sharp corner and a flat side facing the projected trajectory. Several drops are performed for each relative orientation to assure the robustness of the results. From the trajectory analysis we found that the radial dispersion of the particle from the release point increases with increasing $\theta$. However, the increase in radial dispersion is contained within the first section of the trajectory $z<7 D$ and therefore, for vertical positions $z>7 D$ the descent of the particle is not influenced by the initial conditions tested. These results are in accordance with [11], who found that the release angle did not affect the motion mode of the falling disks, but did change the time for the trajectory to reach a saturated state. Thus, in this study the particle trajectory is only analyzed for $z>7 D$. Similarly, the trajectories in this study do not show the last section of the fall to avoid any possible particle interaction with the base of the tank. 
Similarly, each other particle was released 300 times in water at room temperature, $\rho_{f}=$ $0.998 \mathrm{~g} / \mathrm{cm}^{3}$ and $\nu=1.004 \times 10^{-6} \mathrm{~m}^{2} / \mathrm{s}$, the waiting time between drops being of $20 \mathrm{~min}$, corresponding to more than 600 times the particle time-scale of the oscillatory motion.

\section{DETERMINATION OF NON-DIMENSIONAL PARAMETERS AND PHASE DIAGRAM}

Three non-dimensional numbers are considered to be important for the settling dynamics of disks (Chrust et al. 5). They are: dimensionless moment of inertia $\left(I^{*}\right)$, the Galileo number $(G)$ and the particle aspect ratio $(\chi)$. Here, all planar particles are manufactured with $\chi \approx 10$, however, the perimeter of the particles varies with the number of sides. Therefore, this is considered as an important parameter. In order to quantify the perimeter, the isoperimetric quotient $(Q)$ is used where $Q=4 \pi A_{p} / P^{2}$ ( $P$ is the perimeter of the particle). The iso-perimetric inequality, $Q \leq 1$, shows that $Q$ becomes smaller as the particle geometry departs from that of a circular shape as a result of increasing perimeter.

The dimensionless moment of inertia of a disk defined by Willmarth et al. [17] and the Galileo number adopted in Chrust et al. [5] are both based on the disk diameter $(D)$. This definition can not be easily adapted to particles with other frontal geometries and therefore, we re-define these numbers by changing the disk diameter to a length-scale $(L)$

that can be used in conjunction with other geometries, with $L=\sqrt{A_{p}}$. Based on this $L$, the dimensionless moment of inertia is,

$$
I^{*}=\frac{I_{p}}{\rho_{f} L^{5}}
$$

where $I_{p}$ is the mass moment of inertia of the particle and depends on the particle geometry; and the Galileo number,

$$
G=\frac{\sqrt{\left|\rho_{p} / \rho_{f}-1\right| g L^{3}}}{\nu}
$$

This new parameter space allows direct comparison not only of particles with different frontal area but also with severe differences in frontal geometry. Table II shows the three different non-dimensional values for all the particles examined in this study. The Galileo number and dimensionless moment of inertia for all the particles studied in here appear to be in the fluttering regime according to the results shown in Chrust et al. [5].

For the sake of consistency with previous studies, the Reynolds number of the particles 


$\begin{array}{cccccc} & \text { Disk } & \text { Heptagon } & \text { Hexagon } & \text { Pentagon } & \text { Square } \\ Q & 1 & 0.93 & 0.91 & 0.86 & 0.79 \\ I^{*} & 9.33 \times 10^{-3} & 9.19 \times 10^{-3} & 9.18 \times 10^{-3} & 9.25 \times 10^{-3} & 9.55 \times 10^{-3} \\ G & 4195 & 4238 & 4231 & 4238 & 4245\end{array}$

TABLE II: Values of the iso-perimetric quotient $(Q)$, dimensionless moment of inertia $\left(I^{*}\right)$ and Galileo Number $(G)$ of the particles. The length scale $(L)$ used to compute these different non-dimensional numbers was based on $\sqrt{A_{p}}$, where $A_{p}$ is the frontal area of the particles.

(based on the measured average descent velocity) is also computed.

$$
R e=\frac{V_{z} L}{\nu}
$$

where, $V_{z}$ is the mean descent velocity of the particle obtained from the measurements (see next section). This Reynolds number can then be used to identify the space occupied by the planar particles in the $R e-I^{*}$ regime map proposed by Field et al. [8]. Although the introduction of the new length scale $L$ does not significantly change $I^{*}$ of a disk (the length scale is only different by a factor of $\sqrt{\pi} / 2)$, the regime map for disks in the $\left(R e, I^{*}\right)$ domain has to be modified accordingly. Figure 2 shows the regime map defined by Field et al. [8] but re-scaled for the new definition of the dimensionless moment of inertia and the Reynolds number based on $L$. According to the measured Reynolds numbers and dimensionless moment of inertia, planar polygons should exhibit 'planar zig-zag' motion regardless of their shape. The mean descent velocities and the Reynolds number values are indicated in table III.

\section{RESULTS}

\section{A. Descent Velocity}

Figure 3a shows the variation of the measured mean terminal velocity of all planar particles considered in this study. The mean terminal velocity is shown relative to that of the reference disk. The different particles are represented with their iso-perimetric quotient $(Q)$. 


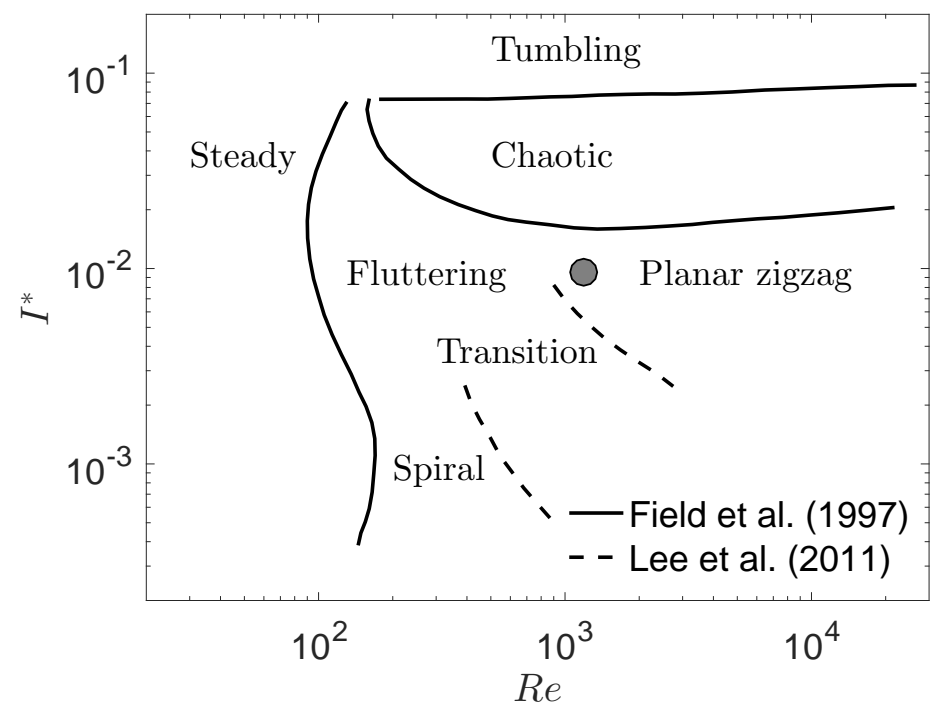

FIG. 2: Phase diagram spanned by the dimensionless moment of inertia $\left(I^{*}\right)$ and Reynolds number $(R e)$. The parameter space has been adapted to account for the change in the definition of the dimensionless numbers. The circle corresponds to the region of the present work. $\left(R e \approx 1200, I^{*} \approx 9 \times 10^{-3}\right)$.

Disk Heptagon Hexagon Pentagon Square

$\begin{array}{lccccc}V_{z}\left(\mathrm{~mm} \mathrm{~s}^{-1}\right) & 58 \pm 3 & 57 \pm 3 & 56 \pm 3 & 56 \pm 3 & 55 \pm 3 \\ \Delta V_{z}\left(\mathrm{~mm} \mathrm{~s}^{-1}\right) & 85 & 81 & 76 & 74 & 57 \\ R e_{L} & 1203 & 1185 & 1168 & 1191 & 1150\end{array}$

TABLE III: Values of mean descent velocity $\left(V_{z}\right)$, peak to peak value of the descent velocity along the trajectory $\left(\Delta V_{z}=V_{z_{\text {min }}}-V_{z_{\text {max }}}\right)$ and Reynolds number. These values are computed based on 300 independent realisations.

It can be seen that as $Q$ increases the terminal velocity of the particle starts to approach the terminal velocity of the disk. Regardless, the variation across all particles tested here is less than $10 \%$. This mean descent velocity $V_{z}$ was used in conjunction with the length scale $L$ to estimate the particle Reynolds number in the previous section. The greatest difference in the mean descent velocity is been found in squares - the particles with the smallest isoperimetric quotient - which is approximately $7 \%$ slower than disks. The magnitude of the velocity difference is in good agreement with the trend shown in Jayaweera [10] for disks and 
a)

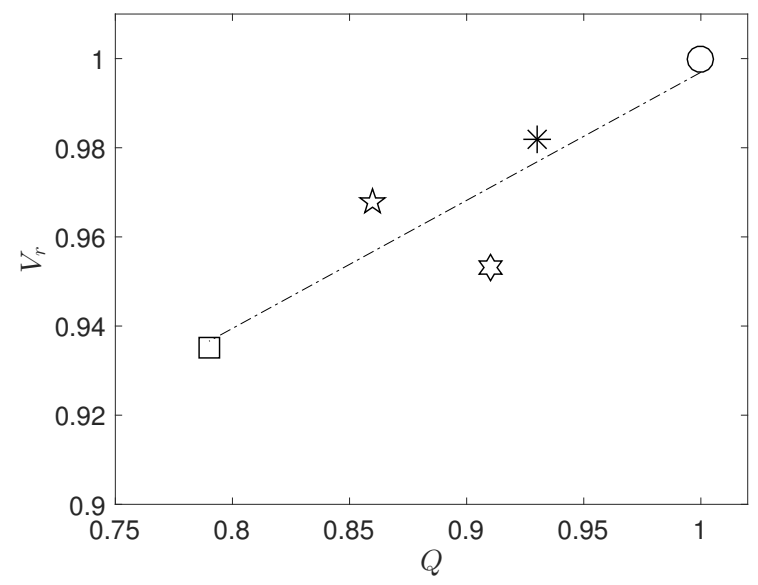

b)

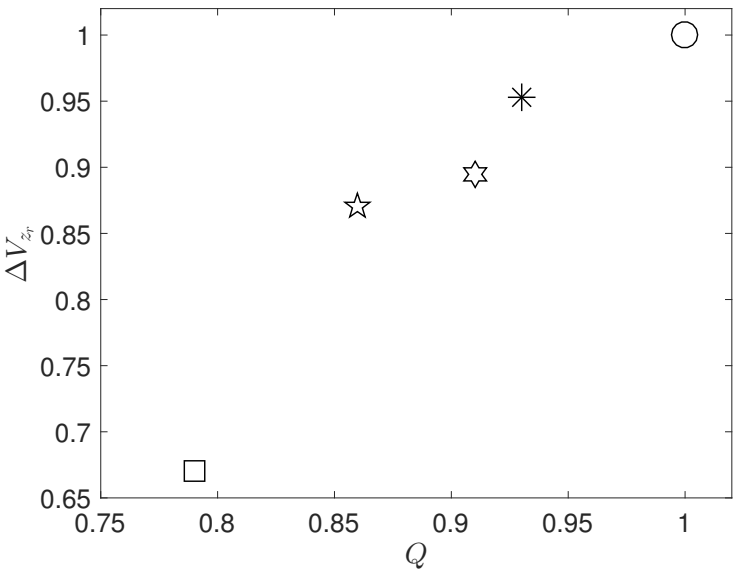

FIG. 3: Dependency of the particle velocity ratio $V_{r}$ and particle peak to peak descent velocity ratio $\Delta V_{z_{r}}$ to the iso-perimetric quotient $Q$.

hexagonal plates covering the range $0.2<R e<22$. However, the particles in Jayaweera [10] are in the steady regime and secondary motion is absent. This suggests that the correlation of shape and descent velocity is similar across the entire fall independent of the flutter or steady regime.

In addition to the mean descent velocity, the peak to peak value of the descent velocity along the trajectory is also computed and the ratio between these and the one for disks $\left(\Delta V_{z_{r}}\right)$ is shown in figure 3b. The peak to peak value of the descent velocity is maximum for disks, and again it reduces with the $Q$, see table III. Squares show a peak to peak velocity value more than $30 \%$ smaller than disks, so that the descent motion of these particle is much steadier than disks. These results are in good agreement with List and Schemenauer [12], where at $R e \approx 200$ small oscillations were observed in disks, hexagonal plates and broad-branched models, with the biggest oscillations present in disks.

The decrease of $\sigma_{r}$ with $Q$ along individual trajectories suggests that the trajectories become less similar to the flutter of a disk as the particle frontal area becomes less axisymmetric. Thus, next section is devoted to the study and classification of the trajectories based on the out-of-plane motion and particle frontal geometry. 


\section{B. Trajectories}

Particle trajectories are analyzed to identify the relavance of the out-of-plane motion along the descent. Several modes of secondary motion are observed for the same particle falling under the same conditions, as observed in Heisinger et al. [9] for disks of $R e \approx 1200$ and $I^{*} \approx 3 \times 10^{-3}$, corresponding to a Galileo number $G \approx 5600$. We find that these modes are not equally probable but depend on the particle geometry. A set of 300 drops per particle is performed to obtain statistic evidence of the influence of the particle geometry on the occurrence of a given secondary motion.

Figure 4(a) shows a 'planar zig-zag' motion while 4(b) shows a trajectory with a strong out-of-plane motion along the particle descent, labelled 'spiral' motion. It is also observed that in some instances the particle descends with severe out-of-plane motion and much steadier descent velocity; and then transitions to a 'planar zig-zag' motion with strong velocity oscillations, as seen in figure 5. This motion transition can also occur from 'spiral' to 'planar zig-zag' motion. This zig-zag-spiral-zig-zag transition coincides with the trajectory pattern in Lee et al. [11] for disks with Reynolds number in the range of $1000-2100$. The planar $(X-Y)$ projection added on the right hand side of the $3 \mathrm{D}$ trajectory shows the relevance of the out-of-plane motion during the fall. Open and closed dots are added to the particle trajectory where the descent velocity is minimum and maximum respectively. As it can be seen, these points define two subsections along the particle path for the "planar zig-zag' motion: a 'gliding' section and a 'turning' section. For 'spiral' motion, figure 4(b), minimum and maximum velocity points appear to be distributed almost randomly relative to the subsections in the 'planar zig-zag' case.

These two modes of motion; i.e 'planar zig-zag' and 'spiral', have been observed in previous experimental and numerical works Chrust et al. [5], Zhong et al. [18] for disks with different dimensionless moment of inertia. However, in this study the same particle describes both types of motion, as seen in Heisinger et al. [9], Lee et al. [11], although they are not equally probable.

Due to the differences observed in the trajectories, a method to classify them according to the relevance of the out-of-plane motion is proposed. To do so, each trajectory is split into sections of gliding motion and of turning motion. These trajectory 'sections' are defined by points of local minimum and maximum descent velocity, open and closed dots in figure 
a)

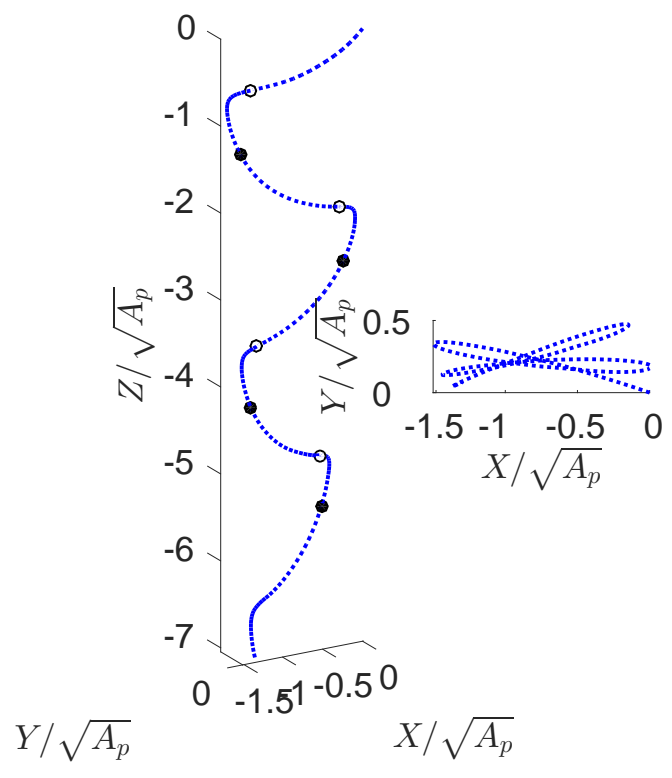

b)

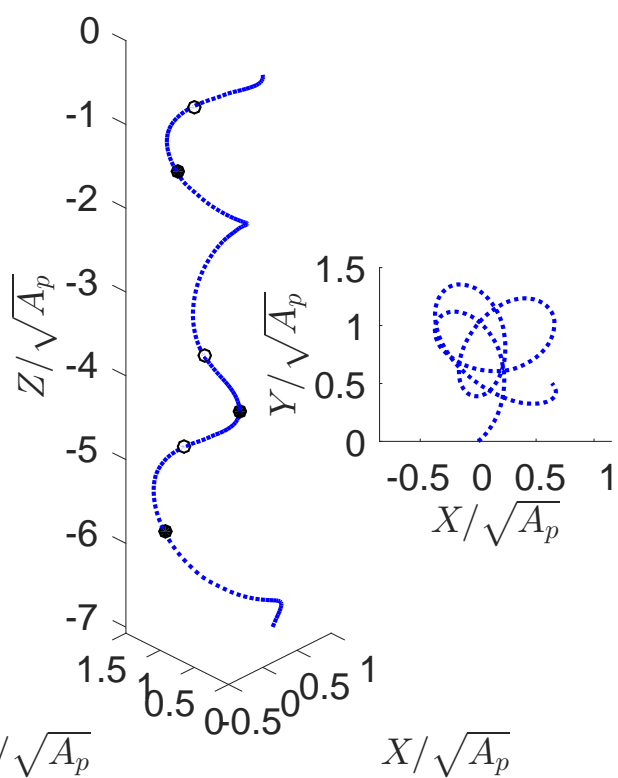

FIG. 4: Reconstructed 3D trajectory sections and (X,Y) planar projections for a disk in $a$ ) 'planar zig-zag' motion, b) 'spiral' motion. Open and closed dots show the location of minimum and maximum descent velocity points respectively.

4.

From the observation of the planar $(X-Y)$ view of any trajectory describing 'planar zigzag' motion, it seems that the gliding sections might be well approximated to a straight line in the $(X-Y)$ plane. Likewise, the turning sections show a complete turn of approximately 180 degrees in the $(X-Y)$ plane. In order to measure the curvature of the trajectory in the $X-Y$ plane during the gliding section, a circle is fitted to the trajectory points, as in figure 6. Then, the distance from the start to the end of the gliding section $(a)$ is compared with the radius $\left(R_{g}\right)$. If the particle describe a 3D spiral motion, the planar $(X-Y)$ view is represented as a circle, and therefore $a / R_{g}=2$. However, if the particle describes a perfect planar motion, the planar $(X-Y)$ view is represented as a straight line, then the radius of the fitted circumference becomes $R_{g}=\infty$, and therefore $a / R_{g}=0$. The value of this ratio could be also zero if the particle falls with zero planar $(X-Y)$ velocity since the amplitude of this motion would be zero. However, this only occurs for particles falling in steady vertical fall. This fitting process can be repeated for each gliding section along the particle trajectory and a mean value of $a / R_{g}$ is obtained per trajectory. This new parameter 


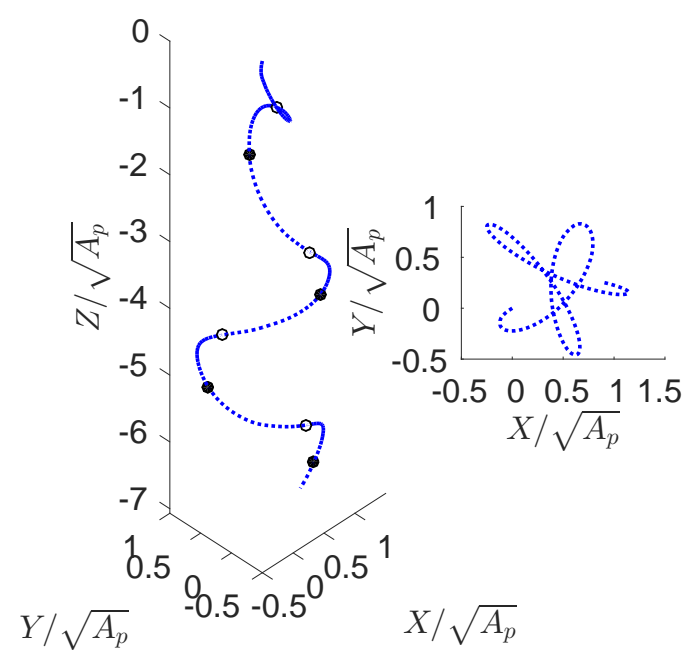

FIG. 5: Reconstructed 3D trajectory section and (X,Y) planar projection for a disk in transition from 'spiral' motion to 'planar zig-zag' fluttering.

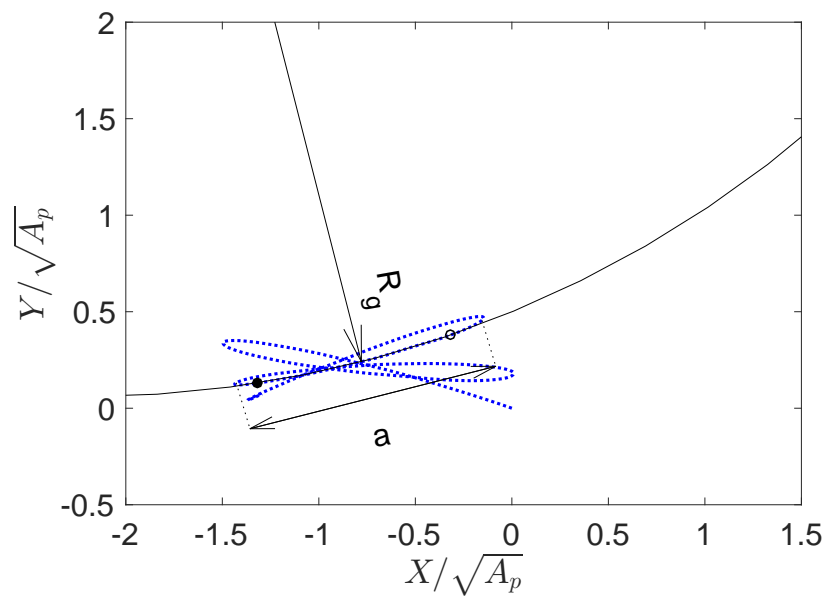

FIG. 6: Schematics of the process to obtain the $\left(a / R_{g}\right)$ ratio of each gliding section.

is used to classify the trajectory followed by the different particles.

In figure 7 a), the cumulative probability of $a / R_{g}$ for different particles is shown. Although $a / R_{g}$ could reach a maximum value of 2 , figure 7 a) shows that experimental data only attains values $a / R_{g} \leq 1$. There is not a significant difference in the statistics above $a / R_{g} \approx 1$. This figure shows that the a very high cumulative probability of nearly 0.8 is achieved by circular disks for $a / R_{g} \leq 0.2$. This means that the disks maintain a nearly linear and planar motion (i.e. planar zig-zag motion). The figure also shows that the heptagons also follow the same trend. Therefore, it can be concluded that the type of trajectory followed by heptagons is 
a)

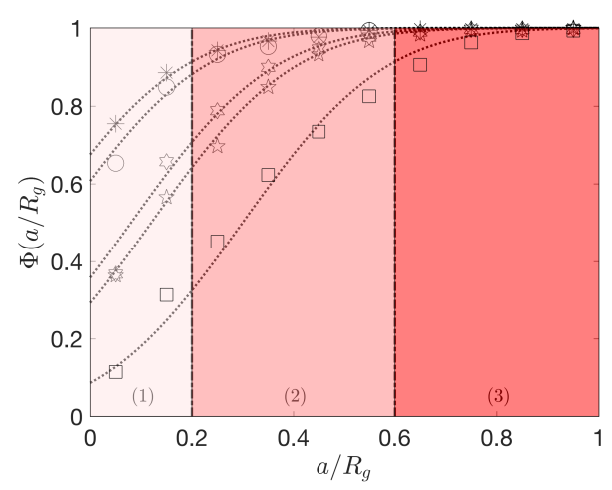

b)

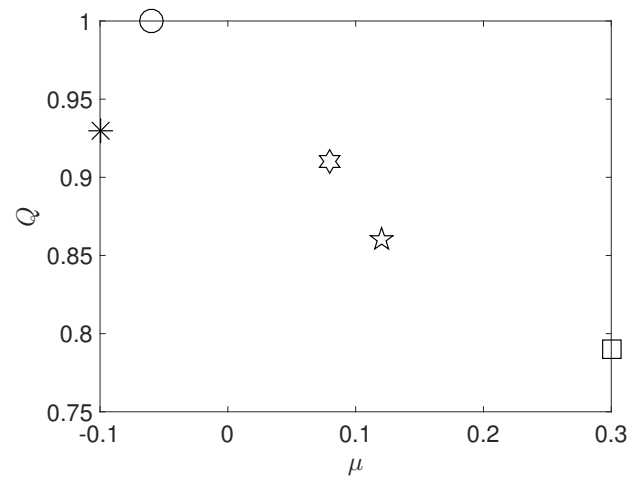

FIG. 7: Cumulative distribution function for the amplitude to radius ratio for each gliding trajectory divided according to the sub-groups proposed; (1) 'planar zig-zag' motion, (2) 'transition' motion and (3) 'spiral' motion in a). Dependency of the fitted constant $\mu$ with the particle iso-perimetric quotient $Q$ in b)

similar to that of disks. It can also be seen that the cumulative probability depends on the iso-perimetric quotient, $Q$, of the particle, as the number of edges in the geometry reduces (as $Q$ decreases), the cumulative probability for $a / R_{g}=0.2$ reduces. This means that the tendency to exhibit 'planar zig-zag' motion (i.e. small values of $a / R_{g}$ ) decreases with $Q$. We believe that the physical reason for the n-polygon particles to deviate from a "planar zig-zag' motion is the existence of an asymetric lift distribution on the particle surface; as explained in [11] for disks, and that this is enhanced as the particle axisymmetry is reduced. We think that the asymmetric lift distribution is due to the misalignment in between the particle motion and any given particle symmetry plane and that leads to a change in the particle orientation with the incoming flow. At the same time, every time that the particle planar motion is reversed the particle orientation relative to the incoming flow is abruptly changed, causing further deviation from the 'planar zig-zag' behaviour. Thus, one can observe that squares (the least axisymmetric particles) only reach a cumulative probability of 0.8 for $a / R_{g} \approx 0.6$ while particles with higher values of $Q$ (and more symmetry planes) have a greater tendency to describe descend motions contained in individual planes. Based on these observations, it is possible to separate tendencies of the trajectory of different particles based on a threshold on $a / R_{g}$. We propose the following classification:

- 'Planar zig-zag' motion $\left[a / R_{g} \leq 0.2\right]$ : The gliding sections are well defined by a straight 
a)

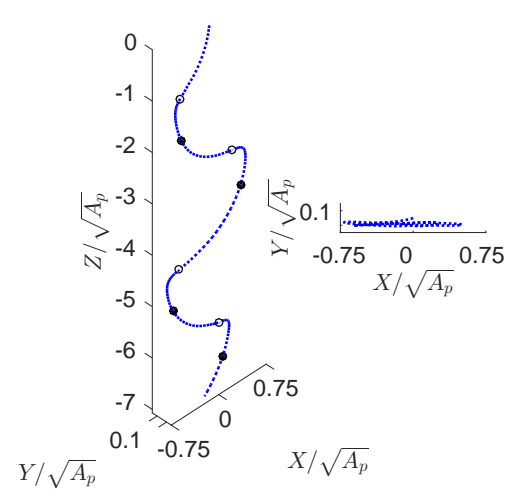

b)

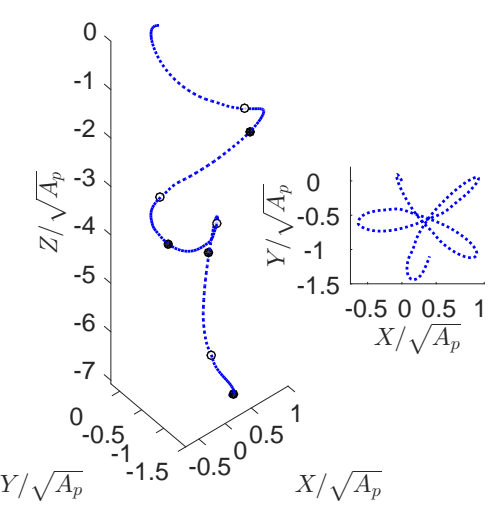

c)

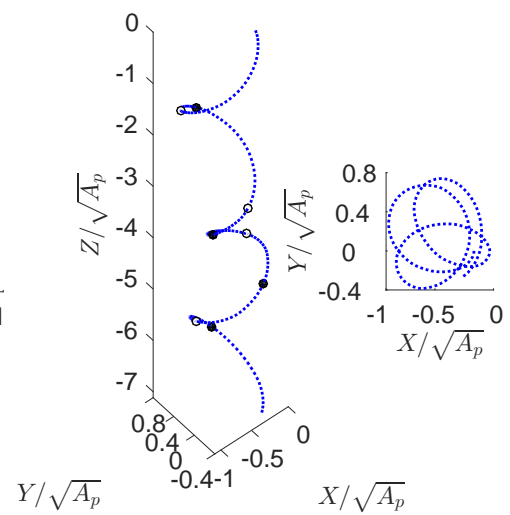

FIG. 8: Sample trajectories of the three sub-regimes proposed: a disk following a "planar zig-zag' motion (a), a pentagon a 'transition' motion (b) and a square a 'spiral' motion (c). Open and closed dots show particle positions of minimum and maximum descent velocity respectively.

line within a $(X-Y)$ plane, while the turning sections show small deviations from the idealised complete turn of 180 degrees in the $(X-Y)$ plane.

- 'Transition' motion $\left[0.2<a / R_{g} \leq 0.6\right]$ : The particle descends following a oscillatory motion in a $(X-Y)$ plane, but with a superimposed constant angular velocity about the z-axis. The particle describes a $(X-Y)$ motion as the bob in a Foucault pendulum seen from a co-rotating point of view.

- 'Spiral' motion $\left[a / R_{g}>0.6\right]$ : There is no clear difference between trajectory sections. The complete trajectory is characterised by a severe and nearly constant out-of-plane motion. This fact results in a much more steady descent velocity.

Figure 8 shows sample trajectories of three different particles that follow the motions described in the above classification. Based on the trajectory classification, it can be seen that disks predominantly follow the 'planar zig-zag' motion, which is consistent with observations in previous studies. As $Q$ decreases, the particle tend to increase the out-of-plane motion and for squares (with the lowest $Q$ ) the motions are predominantly in the 'transition' regime.

The cumulative probability of the particle trajectories can be modelled using the following 
equation,

$$
\Phi(\zeta)=\frac{1}{2}\left(1+\operatorname{erf}\left(\frac{\zeta-\mu}{\sigma \sqrt{2}}\right)\right)
$$

Where $\zeta=a / R_{g}, \sigma$ is a constant (and is equal to 0.22 ) and $\mu$ changes with the particle. The change in $\mu$ with $Q$ is illustrated in figure $7 \mathrm{~b})$. The function $\Phi(\zeta)$ defines the cumulative distribution of a Gaussian distribution with mean $\mu$ and standard deviation $\sigma$. For the measured data, the values of $\mu$ and $\sigma$ that best fit the curves of each planar particle reasonably match the mean and standard deviation of the data, suggesting that the distribution of $a / R_{g}$ is nearly Gaussian. The error functions proposed cannot be used to describe the statistics of the particle motion for $a / R_{g}<0.1$ since they clearly depart from the experimental data at $a / R_{g}=0$, as show the broken lines in figure 7 a).

\section{Revisiting the Phase Diagram}

Disks and heptagons considered in this study show a strong tendency to fall following a 'planar zig-zag' trajectory. However, as the iso-perimetric quotient becomes smaller the out-of-plane motion of the particle gains relevance. This fact shows that the particle frontal geometry has a severe influence in the likeliness of the particle to describe one motion or another. This might be closely related to the likeliness of having a sharp corner aligned with the direction of the particle secondary motion and the resulting wake characteristics. When this occurs, the lift distribution on the particle surface is symmetric with respect to the plane of the secondary motion, and therefore the particle follows a nearly straight line during the gliding section of the trajectory (similar to the motion of a delta wing). Therefore, the deviation from the 'planar zig-zag' motion is caused by flow disturbances that induce the particle rotation about the axis perpendicular to its surface, as detailed in Lee et al. [11]. When the particle symmetry axis is not aligned with the secondary motion, the lift distribution on the particle surface lacks symmetry, thereby inducing the particle rotation and enhancing the transition to 'spiral' motion. Thus, as the frontal geometry changes and the iso-perimetric quotient becomes smaller, the out-of-plane motion of the particle gains relevance and the overall descent becomes similar to the one seen for disks with much smaller dimensionless moment of inertia $\left(I^{*} \approx O\left(10^{-4}\right)\right)$, as seen in Lee et al. [11]. This suggests that the equivalent disks to these other polygons will in fact be larger in diameter.

The differences observed between the trajectories of the particles investigated in here 
Disk Heptagon Hexagon Pentagon Square

$\begin{array}{lccccc}\text { Represented as } & \bigcirc & * & \text { है } & \text { 斿 } & \square \\ I_{L^{\prime}}^{*} & 5.01 \times 10^{-3} & 2.42 \times 10^{-3} & 1.95 \times 10^{-3} & 1.18 \times 10^{-3} & 5.13 \times 10^{-4} \\ R e_{L^{\prime}} & 1370 & 1450 & 1450 & 1540 & 1620\end{array}$

TABLE IV: Particle dimensions. $L^{\prime}$ refers to the new length scale proposed.

a)

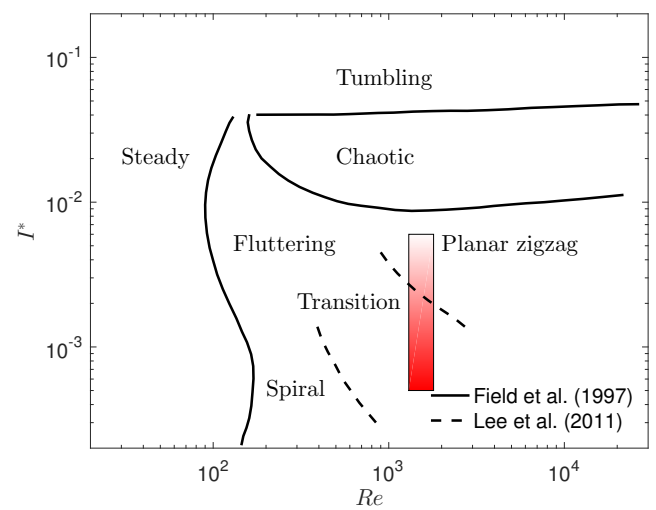

b)

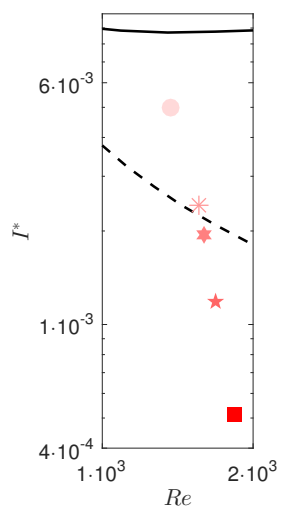

FIG. 9: Phase diagram spanned by the dimensionless moment of inertia $\left(I^{*}\right)$ and Reynolds number $(R e)$. The rectangle corresponds to the region of the present work and the color represents the likeliness of the trajectory to be 'planar zig-zag' (light red) or 'spiral' (dark red),(a). Zoom in the region of the present work (b).

suggest that a characteristic length based on the frontal area of the particle does not define the dimension of equivalent disk to estimate the dynamics of the particle under free fall conditions. We hypothesise that there are two geometric factors affecting the particle motion: the longest distance between any two points along the perimeter of the planar particle and the presence of sharp corners. Both these physical parameters should be accounted for when determining the equivalent disk.

Based on our empirical data and the above-mentioned hypothesis, we propose a new length scale for the estimation of the dimensionless moment of inertia and Reynolds number. This length scale should be related to the diameter of the circumscribed circle around the particle $\left(D_{c}\right)$. This will ensure that the equivalent disk encompasses the entire planar particle and account for the 'longest distance' in our hypothesis. However, this $D_{c}$ is not 
sufficient as the perimeter of the geometry is crucial because the edges add additional vorticity to the field. This edge effect can be accounted for through the iso-perimetric quotient $(Q)$. Therefore, a new length scale $\left(L^{\prime}\right)$ is proposed as ratio between the diameter of the circumscribed circle and the iso-perimetric quotient. This new characteristic length scale, $L^{\prime}=D_{c} / Q$, will make the n-polygons have a much smaller dimensionless moment of inertia and a slightly different Reynolds number, which will alter the phase diagram. More importantly, this new definition reverts to the standard dimensionless inertia and Reynolds number of the disk and is consistent with classical definition used in previous studies.

This length scale $\left(L^{\prime}\right)$ is used to estimate the equivalent dimensionless inertia and particle Reynolds number as seen in table IV. The $\left(R e-I^{*}\right)$ phase diagram based on this new definition for length scale is computed and compared to the observations. First of all, it should be noted that the only change in this revised definition is that the different particles will have a different value of $I^{*}$ depending on their shape (and the corresponding $L^{\prime}$ ). The Reynolds number will also be slightly different (as it depends on $L^{\prime}$ and $V_{z}$ ). Since the values of $I^{*}$ is dramatically different, the different particles now lie in different positions in the phase diagram of a circular disk. This is depicted in figure 9. It can be seen now that the squares lie well in to the 'transition' sub-regime (from 'planar zig-zag' to 'spiral'). The other polygon particles, with higher values of $Q$ are distributed more evenly in the 'transition' sub-regime. Finally, disks are still located in the 'planar zig-zag' sub-regime. These revised locations of the different particles in the phase diagram based on the new length scale agrees very well with the characteristics of the trajectories found in previous sections where we used $a / R_{g}$ to classify the type of trajectory followed by the particles. Based on this new definition almost all particles are in the 'transition' regime (except circular disks and heptagons) with particles with smaller number of edges having a tendency towards spiral motion.

\section{CONCLUSION}

This work investigated the effect of the particle edge geometry on the free falling motion of a planar particle. The reference particle was chosen to be a circular disk that is known to exhibit a 'planar zig-zag' motion (for the values of dimensionless moment of inertia and Reynolds number of the chosen particle). The Galileo number of the different planar particles were held a constant and the iso-perimetric quotient (a measure of particle edge - perimeter) 
was varied by altering frontal geometry of the particle to different n-sided polygons. This allowed us to study the influence of particle shape (or edge) on the descent characteristics of the different planar particles with similar dimensionless inertia and Galileo number.

It was found that mean descent velocity of the planar particles depend on the isoperimetric quotient. Although the magnitude of the mean descent velocity reduces as the perimeter increases, all velocities are within $10 \%$ of the disk mean descent velocity. The peak to peak $\left(V_{z_{\min }}-V_{z_{\max }}\right)$ value in descent velocity was found to decrease with decreasing isoperimetric quotient and variation between circular disks to squares is over $30 \%$. Disks and heptagons were found to describe 'planar zig-zag' trajectories most of the time. This planar motion is characterised by a gliding phase followed by a turning phase. The probability of following a 'planar zig-zag' trajectory decreases with decreasing value of iso-perimetric quotient. The square particles predominantly follows a three-dimensional trajectory; i.e. 'transition' and 'spiral' motion. Pentagons and hexagons exhibit both 'planar zig-zag' and three-dimensional motions with a marginally higher probability of 'spiral' motion for the pentagons compared to hexagons. This firmly addresses one of the questions posed in the review of Moffatt [13]. That is, the edge geometry does indeed alter the characteristics of descent motion.

Based on the observations, a new length scale $\left(L^{\prime}=D_{c} / Q\right)$, is proposed to depend on the circumscribed circle $\left(D_{c}\right)$ and the iso-perimetric quotient $(Q)$, to determine an equivalent disk for these different particles. Comparison of the $R e-I^{*}$ phase diagram for circular disks in the literature to this new equivalent disk show that these equivalent disks with diameter $L^{\prime}$ would undergo the same secondary motions exhibited by the different planar particles. This suggests that it might be possible to reconcile the effects of particle shape using an equivalent disk as long the increased perimeter length of the particles are accounted for in determining the equivalent disk. An important future step would be to confirm the validity of this new length scale for particles of different sizes and also with complex shapes such as fractal edges and for particles lying within other falling regimes such as chaotic and tumbling. 


\section{ACKNOWLEDGMENTS}

This work was supported by Aquavitrum Ltd., the Leverhulme Trust and the Faculty of Engineering and the Environment of University of Southampton. Pertinent data is available at http://doi.org/10.5258/SOTON/D0443 .

[1] Andersen, A., Pesavento, U., and Wang, Z. J. (2005a). Analysis of transitions between fluttering, tumbling and steady descent of falling cards. J. Fluid Mech, 541:91-104.

[2] Andersen, A., Pesavento, U., and Wang, Z. J. (2005b). Unsteady aerodynamics of fluttering and tumbling plates. J. Fluid Mech, 541:65-90.

[3] Auguste, F., Magnaudet, J., and Fabre, D. (2013). Falling styles of disks. J. Fluid Mech., 719:388-405.

[4] Belmonte, A., Eisenberg, H., and Moses, E. (1998). From flutter to tumble: intertial drag and froude similarity in falling paper. Phys. Rev. Lett., 81:345-348.

[5] Chrust, M., Bouchet, G., and Dusek, J. (2013). Numerical simulation of the dynamics of freely falling discs. Physics of Fluids, 25:044102.

[6] Erni, P., Cramer, C., Marti, I., Windhad, E. J., and Fisher, P. (2009). Continuous flow structuring of anisotropic biopolymer particles. Adv. Colloid Interface Sci., 150:16-26.

[7] Fernandes, P. C., Risso, P., Ern, P., and Magnaudet, J. (2007). Oscillatory motion and wake instability of freely rising axisymmetic bodies. J. Fluid Mech., 573:479-502.

[8] Field, S. B., Klaus, M., Moore, M. G., and Nori, F. (1997). Chaotic dynamics of falling disks. Nature, 388:252-254.

[9] Heisinger, L., Newton, P., and Kanso, E. (2014). Coins falling in water. J. Fluid Mech., $714: 243-253$.

[10] Jayaweera, K. O. L. F. (1972). An equivalent disc for calculating the terminal velocities of plate-like ice crystals. J. Atmos. Sci., 29:596-597.

[11] Lee, C., Su, Z., Zhong, H., Chen, S., Zhou, M., and Wu., J. (2013). Experimental investigation of freely falling thin disks. part 2. transition of three-dimensional motion from zigzag to spiral. J. Fluid Mech., 42:77-104.

[12] List, R. and Schemenauer, R. S. (1971). Free-fall behaviour of planar snow crystals, conical 
graupel and small hail. J. Atmos. Sci., 28:110-115.

[13] Moffatt, H. K. (2013). Three coins in a fountain. J. Fluid Mech., 720:1-4.

[14] Moffet, R. C. and Prather, K. A. (2009). In-situ measurements of the mixing state and optical properties of soot with implications for radiative forcing estimates. PNAS, 106:11872-77.

[15] Sabban, L. and van Hout, R. (2011). Measurements of pollen grain dispersal in still air and stationary near homogeneous, isotropic turbulence. J. Aerosol Science., 42:867-882.

[16] Smith, E. H. (1971). Autorotating wings: an experimental investigation. J. Fluid Mech, $50: 513-534$.

[17] Willmarth, W. W., Hawk, N. E., and Harvey, R. L. (1964). Steady and unsteady motions and wakes of freely falling disks. Phys. Fluids, 7:197-208.

[18] Zhong, H., Chen, S., and Lee, C. (2011). Experimental study of freely falling thin disks: Transition from planar zigzag to spiral. Physics of Fluids., 23:011702.

[19] Zhou, W., Chrust, M., and Duek, J. (2017). Path instabilities of oblate spheroids. J. Fluid Mech., 833:445-468. 